\title{
Time Dilation in Relativistic Quantum Decay Laws of Moving Unstable Particles
}

\author{
Filippo Giraldi \\ School of Chemistry and Physics, University of KwaZulu-Natal and National Institute for Theoretical Physics (NITheP), \\ Westville Campus, Durban 4001, South Africa \\ Correspondence should be addressed to Filippo Giraldi; giraldi.filippo@gmail.com
}

Received 20 March 2018; Accepted 22 April 2018; Published 29 May 2018

Academic Editor: Marek Nowakowski

Copyright (C) 2018 Filippo Giraldi. This is an open access article distributed under the Creative Commons Attribution License, which permits unrestricted use, distribution, and reproduction in any medium, provided the original work is properly cited. The publication of this article was funded by SCOAP ${ }^{3}$.

The relativistic quantum decay laws of moving unstable particles are analyzed for a general class of mass distribution densities which behave as power laws near the (nonvanishing) lower bound $\mu_{0}$ of the mass spectrum. The survival probability $\mathscr{P}_{p}(t)$, the instantaneous mass $M_{p}(t)$, and the instantaneous decay rate $\Gamma_{p}(t)$ of the moving unstable particle are evaluated over short and long times for an arbitrary value $p$ of the (constant) linear momentum. The ultrarelativistic and nonrelativistic limits are studied. Over long times, the survival probability $\mathscr{P}_{p}(t)$ is approximately related to the survival probability at rest $\mathscr{P}_{0}(t)$ by a scaling law. The scaling law can be interpreted as the effect of the relativistic time dilation if the asymptotic value $M_{p}(\infty)$ of the instantaneous mass is considered as the effective mass of the unstable particle over long times. The effective mass has magnitude $\mu_{0}$ at rest and moves with linear momentum $p$ or, equivalently, with constant velocity $1 / \sqrt{1+\mu_{0}^{2} / p^{2}}$. The instantaneous decay rate $\Gamma_{p}(t)$ is approximately independent of the linear momentum $p$, over long times, and, consequently, is approximately invariant by changing reference frame.

\section{Introduction}

The description of the decay laws of unstable particles via quantum theory has been a central topic of research for decades $[1,2]$. Many unstable particles which are generated in astrophysical phenomena or high-energy accelerator experiments are moving in the laboratory frame of the observer at relativistic or ultrarelativistic velocity. For this reason, plenty of studies have been devoted to formulate the decay laws in terms of relativistic quantum theory. See [1,3-6], to name but a few.

A fundamental subject in the description of the relativistic decays of moving unstable particles is the way the decay laws transform by changing the reference frame. Naturally, it is essential to understand how the decay laws, holding in the rest reference frame of the moving particle, are transformed in the laboratory frame of an observer. The nondecay or survival probability of an unstable particle has been evaluated in $[7,8]$ for nonvanishing and vanishing values of the linear momentum in terms of the mass distribution density (MDD). The condition of vanishing linear momentum, $p=0$, provides the survival probability in the reference frame where the particle is at rest, while the condition of nonvanishing linear momentum, $p>0$, can be referred to the laboratory frame of an observer where the particles move with linear momentum $p$. The effects of the relativistic time dilation in quantum decay laws of moving unstable particles remain a matter of central interest. See [7-17], to name but a few.

As a continuation of the scenario described above, here, we evaluate the survival probability, the instantaneous energy, and the instantaneous decay rate of a moving unstable particle over short and long times for a wide variety of MDDs and for an arbitrary value of the (constant) linear momentum. In light of the short-time transformations of the survival probability according to the relativistic time dilation, we search for further scaling relations in the decay laws which can relate the conditions of nonvanishing and vanishing linear momentum. In this way, we aim to find further descriptions of the ways the decay laws of moving unstable particle transform by changing reference frame.

The paper is organized as follows. Section 2 is devoted to the relativistic quantum decay laws of a general moving 
unstable particle. In Section 3, the survival probability is evaluated over short and long times for an arbitrary value of the linear momentum and a general class of MDD. In Section 4, the transformation of the long-time survival probability is described via a scaling law. Section 5 is devoted to the evaluation of the instantaneous mass and instantaneous decay rate over short and long times. In Section 6, the scaling law, describing the transformation of the survival probability, is interpreted in terms of the relativistic time dilation and of the instantaneous mass of the moving unstable particle. Summary and conclusions are reported in Section 7.

\section{Relativistic Quantum Decay Laws}

An extended and detailed description of the relativistic quantum decay laws of moving unstable particles has been recently provided in $[14,17]$. Following these references, a brief summary of unstable quantum states, of the survival probability, and of the instantaneous energy and decay rate is reported below for the sake of clarity, by adopting the system of units where $\hbar=c=1$. The motion is assumed to be one-dimensional, due to the conservation of the linear momentum $[1,7,8,14-17]$.

In the Hilbert space $\mathscr{H}$ of the quantum states which describe the unstable particle, let the state kets $|m, p\rangle$ be the common eigenstates of the linear momentum $P$ operator and the Hamiltonian $H$ self-adjoint operator, $P|m, p\rangle=p|m, p\rangle$ and $H|m, p\rangle=E(m, p)|m, p\rangle$, for every value of the mass parameter $m$ and of the linear momentum $p$. The mass parameter $m$ belongs to the spectrum of the Hamiltonian which is supposed to be continuous with lower bound $\mu_{0}$, which means $m \geq \mu_{0}$. In the rest reference frame of the moving particle the linear momentum vanishes and the mentioned state kets become $|m, 0\rangle$, while the eigenstates of the Hamiltonian are $E(m, 0)=m$. Let $|\phi\rangle$ be the ket of the Hilbert space $\mathscr{H}$ which describes the quantum state of the unstable particle. Such state can be expressed in terms of the eigenstates $|m, 0\rangle$ of the Hamiltonian as $|\phi\rangle=$ $\int_{\mu_{0}}^{\infty} f(m)|m, 0\rangle d m$, via the expansion function $f(m)$. The survival amplitude $A_{0}(t)$ is defined in the rest reference frame of the moving unstable particle as $A_{0}(t)=\left\langle\phi\left|e^{-\imath H t}\right| \phi\right\rangle$ and is given by the integral expression

$$
A_{0}(t)=\int_{\mu_{0}}^{\infty} \omega(m) e^{-\imath m t} d m,
$$

where $\imath$ is the imaginary unit. The function $\omega(m)$ represents the MDD and reads $\omega(m)=|f(m)|^{2}$. The probability $\mathscr{P}_{0}(t)$ that the decaying particle is in the initial state at the time $t$, i.e., the survival probability, is given by the following form, $\mathscr{P}_{0}(t)=\left|A_{0}(t)\right|^{2}$, in the rest reference frame of the moving unstable particle.

Let $\Lambda$ be the Lorentz transformation which relates the reference frame where the unstable moving particle is at rest, to the one with velocity $v=p /\left(m \gamma_{L}\right)$, where $\gamma_{L}$ is the corresponding relativistic Lorentz factor. Let $U(\Lambda)$ be an unitary representation of the transformation $\Lambda$ acting on the Hilbert space $\mathscr{H}$ such that $|m, p\rangle=U(\Lambda)|m, 0\rangle$ for every value of the mass parameter $m$ in the Hamiltonian spectrum.
The state ket $|\phi, p\rangle$ describes the moving unstable particle with nonvanishing linear momentum $p$. Such state is related to the state ket $|\phi\rangle$ as follows: $|\phi, p\rangle=U(\Lambda)|\phi\rangle$. The form $E(m, p)=m \gamma_{L}=\sqrt{p^{2}+m^{2}}$ is obtained by considering the energy-momentum 4 -vector and the Lorentz invariance [6, 18]. The quantity $A_{p}(t)$ is defined as $A_{p}(t)=\left\langle p, \phi\left|e^{-\imath H t}\right| \phi, p\right\rangle$ and represents the survival amplitude in the reference frame where the particle has linear momentum $p$. The approach described above leads to the following integral expression of the survival amplitude:

$$
A_{p}(t)=\int_{\mu_{0}}^{\infty} \omega(m) e^{-\imath \sqrt{p^{2}+m^{2}} t} d m .
$$

The quantity $\mathscr{P}_{p}(t)$ represents the survival probability that the decaying particle is in the initial state at the time $t$ in the reference frame where the unstable particle has linear momentum $p$ and is given by the square modulus of the survival amplitude, $\mathscr{P}_{p}(t)=\left|A_{p}(t)\right|^{2}$. See $[7,8,17]$ for details.

The decay laws of the unstable particles are obtained from the MDD via (1) and (2). In literature, the MDD is usually represented via the Breit-Wigner function [19]:

$$
\omega_{\mathrm{BW}}(m)=\frac{\Theta\left(m-\mu_{0}\right) \lambda_{\mathrm{BW}} \bar{\Gamma} /(2 \pi)}{\left(m-m_{0}\right)^{2}+\bar{\Gamma}^{2} / 4},
$$

where $\lambda_{\mathrm{BW}}$ is a normalization factor, $\Theta(m)$ is the Heaviside unit step function, $m_{0}$ is the rest mass of the particle, and $\bar{\Gamma}$ is the decay rate at rest. A detailed analysis of the survival amplitude of a moving unstable particle has been performed in [8] by considering the Breit-Wigner form of the MDD [7]. The long-time behavior of the survival amplitude results in dominant inverse power laws, besides additional decaying exponential terms. Refer to [8] for details. In [17, $20,21]$ general forms of MDD are considered with power-law behaviors near the lower bound of the mass spectrum. The long-time decay of the survival amplitude $A_{0}(t)$ is described by inverse power laws which are determined by the lowmass profile of the $\operatorname{MDD}[20,21]$. Additional removable logarithmic singularities in the low-mass form of the MDD lead to logarithmic-like relaxations of the survival amplitude at rest $A_{0}(t)$ which can be arbitrarily slower or faster than inverse power laws [22, 23].

Since the unstable particle decays, the initial state is not an eigenstate of the Hamiltonian and the instantaneous mass (energy) is not defined during the time evolution. For this reason, instantaneous mass (energy) and decay rate are defined in the rest reference frame of the moving particle in terms of an effective Hamiltonian. Such operator acts on the subspace of the Hilbert space $\mathscr{H}$ which is spanned by the initial state. Refer to $[14,17,20,21,24]$ for details. In the same way, the instantaneous mass (energy) and decay rate $\Gamma_{p}(t)$ of the moving unstable particle are defined in the frame system where the particle has linear momentum $p$. For both vanishing and nonvanishing values of the linear momentum $p$, the instantaneous mass $M_{p}(t)$ and decay rate $\Gamma_{p}(t)$ are 
obtained from the survival amplitude $A_{p}(t)$ via the following forms [14, 17, 20-23]:

$$
\begin{aligned}
& M_{p}(t)=-\operatorname{Im}\left\{\frac{\dot{A}_{p}(t)}{A_{p}(t)}\right\} \\
& \Gamma_{p}(t)=-2 \operatorname{Re}\left\{\frac{\dot{A}_{p}(t)}{A_{p}(t)}\right\} .
\end{aligned}
$$

The long-time behavior of the instantaneous mass $M_{p}(t)$ and decay rate $\Gamma_{p}(t)$ has been evaluated in $[14,15]$ for the BreitWigner form of the MDD.

2.1. Relativistic Time Dilation. The interpretation of decay processes via the theory of special relativity suggests that the lifetime which is detected in the rest reference frame of the unstable particle increases in the reference frame where the particle is moving. The increase is due to the relativistic dilation of times and is determined by the relativistic Lorentz factor [25].

The appearance of the relativistic time dilation in quantum decays is a matter of great interest and fruitful discussions. See [7-17], to name but a few. A broadly shared opinion is that the relativistic time dilation influences the survival probability uniquely in the short-time exponential decay. Briefly, this means that the survival probability $\mathscr{P}_{p}(t)$ and the survival probability at rest $\mathscr{P}_{0}(t)$ are given by the exponential forms $\mathscr{P}_{p}(t) \sim e^{-t / \tau_{p}}$ and $\mathscr{P}_{0}(t) \sim e^{-t / \tau_{0}}$, over short times. Under this assumption, the survival probability obeys the scaling law

$$
\mathscr{P}_{p}(t) \sim \mathscr{P}_{0}\left(\frac{t}{\gamma_{L}}\right),
$$

over short times. The parameter $\tau_{0}$ represents the lifetime of the particle at rest, while $\tau_{p}$ is the lifetime which is detected in the reference frame where the particle is moving with linear momentum $p$. According to the relativistic time dilation, the lifetimes are related as follows: $\tau_{p}=\tau_{0} \gamma_{L}$, where $\gamma_{L}$ is the corresponding relativistic Lorentz factor. Refer to $[7,8,12-$ 17] for an extended explanation. In this context, we intend to study the survival probability $\mathscr{P}_{p}(t)$ and the survival probability at rest $\mathscr{P}_{0}(t)$ over short and long times for a wide variety of MDDs. We search for further ways to describe the transformations of the decay laws which occur by changing reference frame.

\section{Survival Probability versus Linear Momentum}

In the present section the short- and long-time behaviors of the survival probability are studied for a general value $p$ of the constant linear momentum of the moving unstable particle. The analysis performed in the whole paper is based entirely on form (2) of the survival amplitude $[7,12]$. For the sake of convenience, the survival amplitude is expressed via the dimensionless variables $\tau, \xi, \rho$, and $\eta$. These variables are defined in terms of a generic scale mass $m_{s}$ as follows: $\tau=m_{s} t, \xi=m / m_{s}, \rho=p / m_{s}$, and $\eta=\sqrt{\rho^{2}+\xi^{2}}$. The MDD is expressed in terms of the auxiliary function $\Omega(\xi)$ via the scaling law $\omega\left(m_{s} \xi\right)=\Omega(\xi) / m_{s}$, for every $\xi \geq \xi_{0}$, where $\xi_{0}=\mu_{0} / m_{s}$. In this way, the survival amplitude $A_{p}(t)$ results in the expression below:

$$
A_{p}(t)=\int_{\xi_{0}}^{\infty} \Omega(\xi) e^{-\imath \eta \tau} d \xi .
$$

The MDDs under study are defined over the infinite support $\left[\mu_{0}, \infty\right)$ by auxiliary functions $\Omega(\xi)$ of the following form:

$$
\Omega(\xi)=\left(\xi-\xi_{0}\right)^{\alpha} \Omega_{0}(\xi) .
$$

In order to study the long-time behavior of the decay laws of the moving unstable particle, the MDDs are requested to obey the conditions below. The lower bound of the mass spectrum is chosen to be nonvanishing, $\xi_{0}>0$. The constraints $\alpha \geq 0$ and $\Omega_{0}\left(\xi_{0}\right)>0$ are also requested. The function $\Omega_{0}(\xi)$ and the derivatives $\Omega_{0}^{(j)}(\xi)$ are required to be summable, for every $j=1, \ldots,\lfloor\alpha\rfloor+4$, and continuously differentiable in the whole support $\left[\mu_{0}, \infty\right)$, for every $j=1, \ldots,\lfloor\alpha\rfloor+3$. Consequently, the limits $\lim _{\xi \rightarrow \xi_{0}^{+}} \Omega_{0}^{(j)}(\xi)$ must exist as finite and be $\Omega_{0}^{(j)}\left(\xi_{0}\right)$ for every $j=0, \ldots,\lfloor\alpha\rfloor+4$. The functions $\Omega_{0}^{(j)}(\xi)$ have to decay sufficiently fast as $\xi \rightarrow+\infty$, so that the auxiliary function $\Omega(\xi)$ and the derivatives $\Omega^{(j)}(\xi)$ vanish as $\xi \rightarrow+\infty$, for every $j=0, \ldots,\lfloor\alpha\rfloor$.

As far as the short-time behavior of the survival amplitude is concerned, let the auxiliary function decay as follows: $\Omega(\xi)=\mathcal{O}\left(\xi^{-1-l_{0}}\right)$ for $\xi \rightarrow+\infty$, with $l_{0}>5$. Under this condition, the survival amplitude evolves algebraically over short times:

$$
A_{p}(t) \sim 1-\imath a_{0} t-a_{1} t^{2}+\imath a_{2} t^{3}
$$

for $t \ll 1 / m_{s}$. The constants $a_{0}, a_{1}$, and $a_{2}$ are given by the expressions below:

$$
\begin{aligned}
& a_{0}=\int_{\mu_{0}}^{\infty} \omega(m) \sqrt{p^{2}+m^{2}} d m, \\
& a_{1}=\frac{1}{2} \int_{\mu_{0}}^{\infty} \omega(m)\left(p^{2}+m^{2}\right) d m, \\
& a_{2}=\frac{1}{6} \int_{\mu_{0}}^{\infty} \omega(m)\left(p^{2}+m^{2}\right)^{3 / 2} d m .
\end{aligned}
$$

The short-time evolution of the survival probability is derived from (7) and (9) and is algebraic:

$$
\mathscr{P}_{p}(t) \sim 1-\pi_{0} t^{2}
$$

for $t \ll 1 / m_{s}$, where $\pi_{0}=2 a_{1}-a_{0}^{2}$.

The long-time behavior of the survival amplitude is obtained from (7) and from the following equivalent form:

$$
A_{p}(t)=\int_{\eta_{0}}^{\infty} \frac{\eta \Omega\left(\sqrt{\eta^{2}-\rho^{2}}\right)}{\sqrt{\eta^{2}-\rho^{2}}} e^{-\imath \eta \tau} d \eta
$$


where $\eta_{0}=\sqrt{\rho^{2}+\xi_{0}^{2}}$. Notice that the constraint of nonvanishing lower bound of the mass spectrum, $\xi_{0}>0$, is fundamental for the equivalence of expressions (7) and (12) of the survival amplitude. The asymptotic analysis $[26,27]$ of the integral form, appearing in (12) for $\tau \gg 1$, provides the expression of the survival amplitude over long times:

$$
A_{p}(t) \sim c_{0} e^{-\imath\left((\pi / 2)(1+\alpha)+\sqrt{\mu_{0}^{2}+p^{2}} t\right)}\left(\frac{\chi_{p}}{m_{s} t}\right)^{1+\alpha},
$$

for $t \gg 1 / m_{s}$, where $c_{0}=\Gamma(1+\alpha) \Omega_{0}\left(\xi_{0}\right)$, and

$$
\chi_{p}=\sqrt{1+\frac{p^{2}}{\mu_{0}^{2}}} .
$$

Asymptotic form (13) of the survival amplitude holds for every value of the linear momentum $p$, nonvanishing, arbitrarily large or small, or vanishing; for the variety of MDDs under study; for every $\alpha \geq 0$; and for every value $\mu_{0}$ of the lower bound of the mass spectrum such that $\mu_{0} / m_{s}>0$. The last condition is crucial and will be interpreted in the next section in terms of the instantaneous mass at rest of the moving unstable particle. The square modulus of asymptotic expression (13) approximates the survival probability over long times:

$$
\mathscr{P}_{p}(t) \sim c_{0}^{2}\left(\frac{\chi_{p}}{m_{s} t}\right)^{2(1+\alpha)}
$$

for $t \gg 1 / m_{s}$. Notice that the time scale $1 / m_{s}$ and, consequently, the short or long times, $t \ll 1 / m_{s}$ or $t \gg$ $1 / m_{s}$, are independent of the auxiliary function $\Omega(\xi)$ and are determined uniquely by the MDD.

\section{Scaling Law for the Survival Probability}

Expression (15) of the survival probability holds for every value of the constant linear momentum $p$. Such arbitrariness allows evaluating the survival probability in the nonrelativistic and ultrarelativistic limits. For vanishing value of the linear momentum, $p=0$, or, equivalently, in the rest reference frame of the unstable particle, the survival probability is approximated over long times as follows:

$$
\mathscr{P}_{0}(t) \sim \frac{c_{0}^{2}}{\left(m_{s} t\right)^{2(1+\alpha)}},
$$

for $t \gg 1 / m_{s}$. Instead, consider large values of the linear momentum, $p \gg \mu_{0}$. In such condition the survival probability is approximated over long times as follows:

$$
\mathscr{P}_{p}(t) \sim c_{0}^{2}\left(\frac{p}{\mu_{0} m_{s} t}\right)^{2(1+\alpha)},
$$

for $t \gg 1 / m_{s}$. By comparing (15) and (16), we observe that, for the MDDs under study, the survival probability obeys, approximately over long times, the following scaling law:

$$
\mathscr{P}_{p}(t) \sim \mathscr{P}_{0}\left(\frac{t}{\chi_{p}}\right),
$$

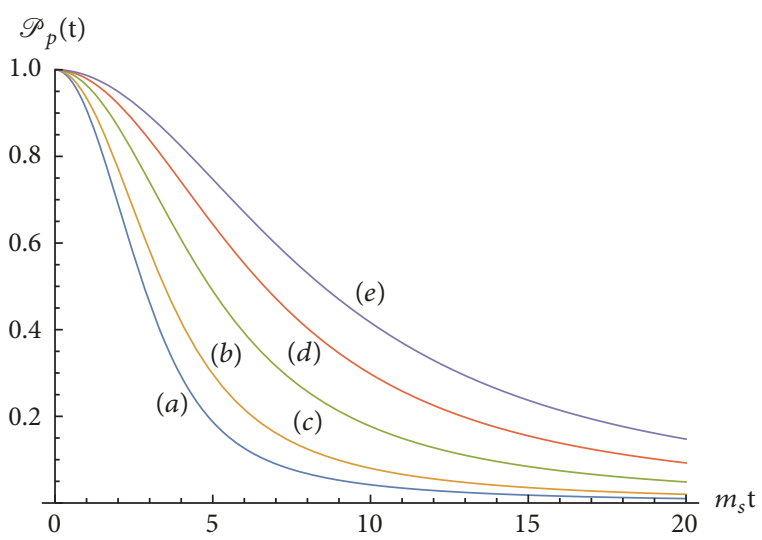

FIGURE 1: (Color online) the survival probability $\mathscr{P}_{p}(t)$ versus $\left(m_{s} t\right)$ for $0 \leq m_{s} t \leq 20$, MDDs given by (21), $\alpha=0, \mu_{0}=m_{s}$, and different values of the linear momentum $p$. Curve $(a)$ corresponds to $p=$ $0 m_{s} ;(b)$ corresponds to $p=m_{s} ;(c)$ corresponds to $p=2 m_{s} ;(d)$ corresponds to $p=3 m_{s} ;(e)$ corresponds to $p=4 m_{s}$.

for $t \gg 1 / m_{s}$. This is the main result of the paper. In fact, the above scaling law describes how the survival probability at rest transforms, approximately over long times, in the reference frame where the particle moves with constant linear momentum $p$. The transformation can be interpreted, approximately, as the effect of a time dilation which is determined by the scaling factor $\chi_{p}$. Notice that the scaling factor, given by (14), diverges in the limit $\mu_{0} \rightarrow 0^{+}$. In Section 6 , the scaling law (18) is interpreted, via the special relativity, as the effect of the relativistic time dilation. This interpretation holds if the asymptotic value of the instantaneous mass is considered as the effective mass of the moving unstable particle over long times.

The correction to the scaling law (18) can be estimated via the expression $\left(\mathscr{P}_{p}(t)-\mathscr{P}_{0}\left(t / \chi_{p}\right)\right) / \mathscr{P}_{0}\left(t / \chi_{p}\right)$. For the MDDs under study, such correction vanishes inversely quadratically over long times:

$$
\frac{\mathscr{P}_{p}(t)-\mathscr{P}_{0}\left(t / \chi_{p}\right)}{\mathscr{P}_{0}\left(t / \chi_{p}\right)} \sim \frac{\kappa_{p}}{\left(m_{s} t\right)^{2}},
$$

for $t \gg 1 / m_{s}$, where

$$
\begin{aligned}
\kappa_{p} & =\frac{(1+\alpha)(2+\alpha) m_{s} p^{2}}{\mu_{0}^{3}}\left(2 \frac{\Omega_{0}^{\prime}\left(\mu_{0} / m_{s}\right)}{\Omega_{0}\left(\mu_{0} / m_{s}\right)}-\frac{m_{s}}{\mu_{0} \chi_{p}^{2}}\right. \\
& \left.\times\left(3+\alpha+\left(\frac{5}{2}+\alpha\right) \frac{p^{2}}{\mu_{0}^{2}}\right)\right) .
\end{aligned}
$$

Numerical analysis of the survival probability $\mathscr{P}_{p}(t)$ has been displayed in Figures 1, 2, 3, 4, and 5. The computed MDDs are given by the following toy form of the auxiliary function:

$$
\Omega(\xi)=w_{\alpha} \xi\left(\xi^{2}-\xi_{0}^{2}\right)^{\alpha} e^{-\xi^{2}}
$$




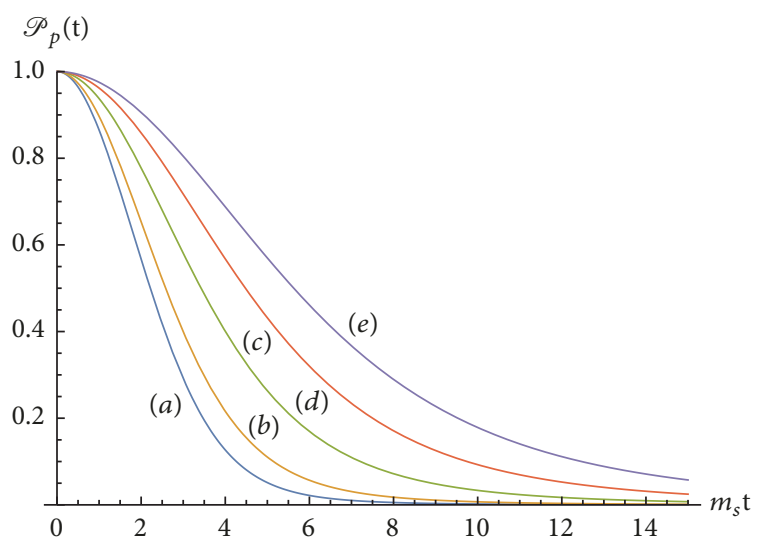

FIGURE 2: (Color online) the survival probability $\mathscr{P}_{p}(t)$ versus $\left(m_{s} t\right)$ for $0 \leq m_{s} t \leq 15$, MDDs given by (21), $\alpha=1, \mu_{0}=m_{s}$, and different values of the linear momentum $p$. Curve $(a)$ corresponds to $p=$ $0 m_{s} ;(b)$ corresponds to $p=m_{s} ;(c)$ corresponds to $p=2 m_{s} ;(d)$ corresponds to $p=3 m_{s} ;(e)$ corresponds to $p=4 m_{s}$.

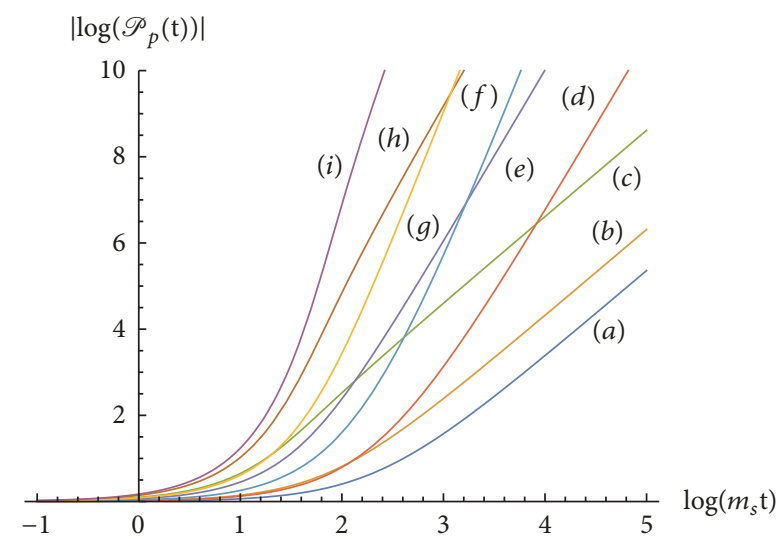

FIGURE 3: (Color online) quantity $\left|\log \left(\mathscr{P}_{p}(t)\right)\right|$ versus $\log \left(m_{s} t\right)$ for $e^{-1} \leq m_{s} t \leq e^{5}$, MDDs given by (21), $\mu_{0}=m_{s}$, and different values of the parameter $\alpha$ and of the linear momentum $p$. Curve (a) corresponds to $\alpha=0$ and $p=5 m_{s} ;(b)$ corresponds to $\alpha=0$ and $p=3 m_{s} ;(c)$ corresponds to $\alpha=0$ and $p=0 ;(d)$ corresponds to $\alpha=1$ and $p=5 m_{s} ;(e)$ corresponds to $\alpha=1$ and $p=2 m_{s} ;(f)$ corresponds to $\alpha=2$ and $p=4 m_{s} ;(g)$ corresponds to $\alpha=2$ and $p=2 m_{s} ;(h)$ corresponds to $\alpha=1$ and $p=0 m_{s} ;(i)$ corresponds to $\alpha=2$ and $p=0$.

where $w_{\alpha}$ is a normalization factor and reads $w_{\alpha}=2 e^{\xi_{0}^{2}} / \Gamma(1+$ $\alpha$ ). Different values of the nonnegative power $\alpha$ and linear momentum $p$ are considered. The corresponding MDDs belong to the class under study which is defined in Section 3 via (8). The asymptotic lines appearing in Figure 3 agree with the long-time inverse-power-law decays of the survival probability, given by (15). The ordinates of the asymptotic horizontal lines of Figures 4 and 5 are in accordance with form (14) of the scaling factor $\chi_{p}$. The long-time dilation in the survival probability, given by the scaling law (18), is confirmed by the common horizontal asymptotic line, at ordinate 1, which appears in Figures 6 and 7.

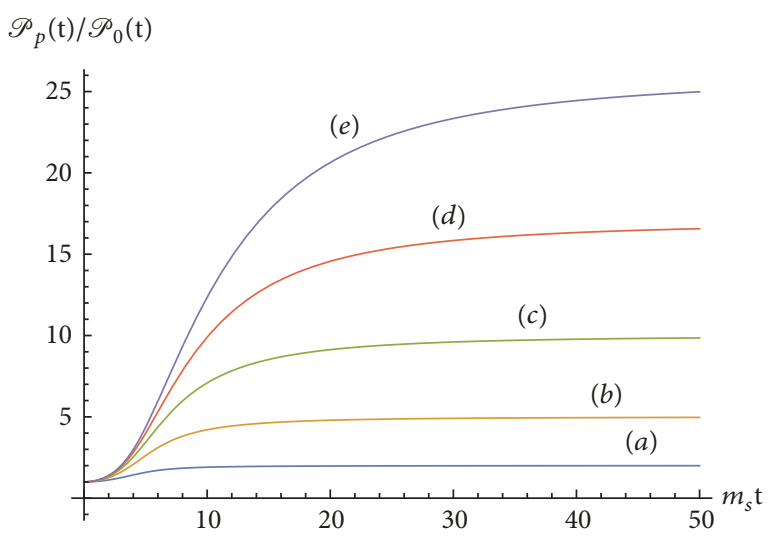

Figure 4: (Color online) ratio $\mathscr{P}_{p}(t) / \mathscr{P}_{0}(t)$ versus $\left(m_{s} t\right)$ for $0 \leq$ $m_{s} t \leq 50$, MDDs given by (21), $\mu_{0}=m_{s}, \alpha=0$, and different values of the linear momentum $p$. Curve $(a)$ corresponds to $p=m_{s}$; (b) corresponds to $p=2 m_{s} ;(c)$ corresponds to $p=3 m_{s} ;(d)$ corresponds to $p=4 m_{s} ;(e)$ corresponds to $p=5 m_{s}$.

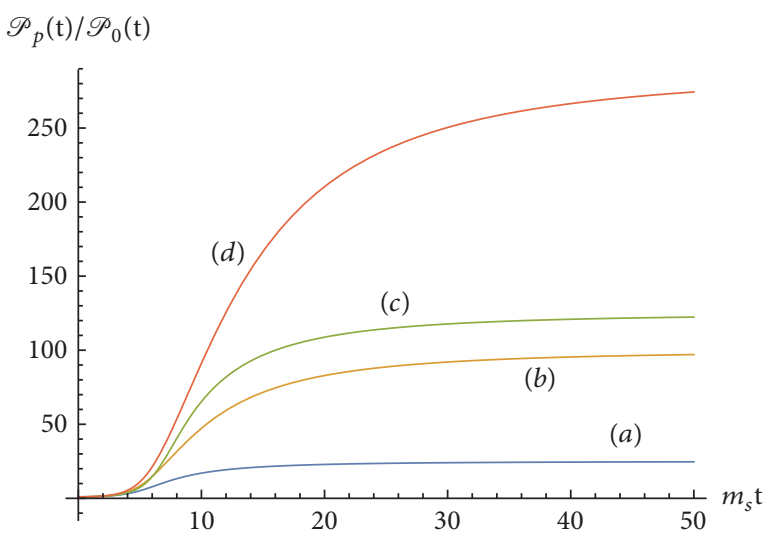

Figure 5: (Color online) ratio $\mathscr{P}_{p}(t) / \mathscr{P}_{0}(t)$ versus $\left(m_{s} t\right)$ for $0 \leq$ $m_{s} t \leq 50$, MDDs given by (21), $\mu_{0}=m_{s}$, and different values of the parameter $\alpha$ and of the linear momentum $p$. Curve (a) corresponds to $\alpha=1$ and $p=2 m_{s} ;(b)$ corresponds to $\alpha=1$ and $p=3 m_{s} ;(c)$ corresponds to $\alpha=2$ and $p=2 m_{s} ;(d)$ corresponds to $\alpha=1$ and $p=4 m_{s}$.

\section{Instantaneous Mass and Decay Rate versus Linear Momentum}

In the present section the instantaneous mass and decay rate are analyzed over short and long times for every value of the constant linear momentum $p$ of the particle, which is detected in the laboratory frame of the observer. The instantaneous mass and decay rate are evaluated from the survival amplitude via (4) and (5).

Again, let the auxiliary function decay as $\Omega(\xi)=\mathcal{O}\left(\xi^{-1-l_{0}}\right)$ for $\xi \rightarrow+\infty$, with $l_{0}>5$. The short-time evolution of the instantaneous mass and decay rate are obtained from behavior (9) of the survival amplitude and from (4) and (5). In this way, the following algebraic evolution results over short times: 


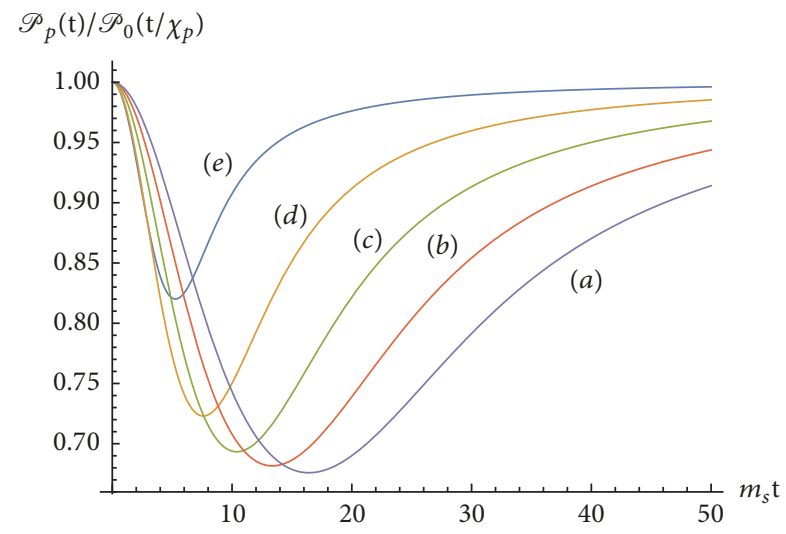

Figure 6: (Color online) ratio $\mathscr{P}_{p}(t) / \mathscr{P}_{0}\left(t / \chi_{p}\right)$ versus $\left(m_{s} t\right)$ for $0 \leq$ $m_{s} t \leq 50$, MDDs given by (21), $\mu_{0}=m_{s}, \alpha=0$, and different values of the linear momentum $p$. Curve $(a)$ corresponds to $p=5 m_{s}$; (b) corresponds to $p=4 m_{s} ;(c)$ corresponds to $p=3 m_{s} ;(d)$ corresponds to $p=2 m_{s} ;(e)$ corresponds to $p=m_{s}$.

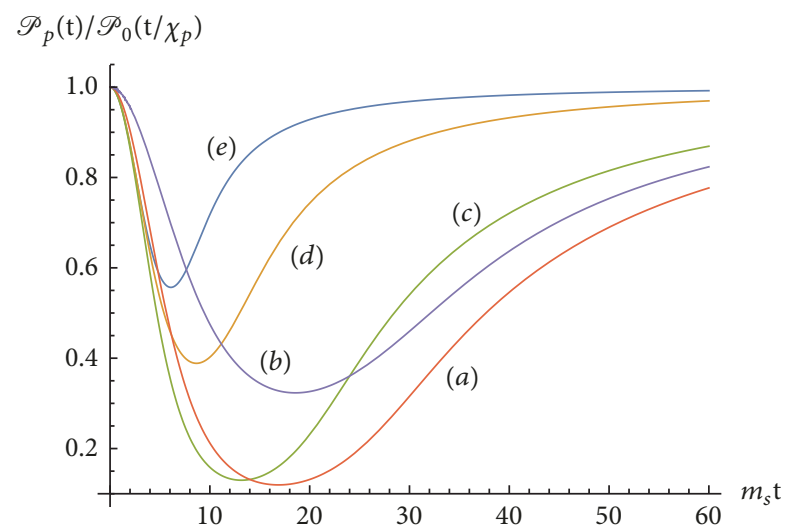

Figure 7: (Color online) ratio $\mathscr{P}_{p}(t) / \mathscr{P}_{0}\left(t / \chi_{p}\right)$ for $0 \leq m_{s} t \leq 60$, MDDs given by (21), $\mu_{0}=m_{s}$, and different values of the parameter $\alpha$ and of the linear momentum $p$. Curve (a) corresponds to $\alpha=2$ and $p=4 m_{s} ;(b)$ corresponds to $\alpha=1$ and $p=5 m_{s} ;(c)$ corresponds to $\alpha=2$ and $p=3 m_{s} ;(d)$ corresponds to $\alpha=1$ and $p=2 m_{s} ;(e)$ corresponds to $\alpha=1$ and $p=m_{s}$.

$$
\begin{aligned}
M_{p}(t) & \sim a_{0}-\pi_{1} t^{2}, \\
\Gamma_{p}(t) & \sim \pi_{2} t,
\end{aligned}
$$

for $t \ll 1 / m_{s}$, where $\pi_{1}=a_{0}^{3}+3\left(a_{2}-a_{0} a_{1}\right)$ and $\pi_{2}=2\left(2 a_{1}-a_{0}^{2}\right)$.

The long-time behavior of the instantaneous mass and decay rate are studied in case that the MDD fulfills the constraints which are reported in the second paragraph of Section 3. In addition, the functions $\xi \Omega_{0}(\xi)$ and $\xi \Omega(\xi)$ are required to obey the conditions which are requested in the same paragraph for the function $\Omega_{0}(\xi)$ and the auxiliary function $\Omega(\xi)$, respectively. The asymptotic analysis $[26,27]$ of the instantaneous mass or the instantaneous decay rate is obtained from the results of Section 3 and from (4) or (5), respectively.
The instantaneous mass tends over long times, $t \gg 1 / m_{s}$, to the asymptotic value $M_{p}(\infty)$, given by

$$
M_{p}(\infty)=\sqrt{\mu_{0}^{2}+p^{2}}
$$

according to the following dominant algebraic decay:

$$
M_{p}(t) \sim M_{p}(\infty)\left(1+\zeta_{p}\left(m_{s} t\right)^{-2}\right) .
$$

The constant $\zeta_{p}$ is defined as

$$
\begin{aligned}
\zeta_{p}= & (1+\alpha) \\
& \cdot \frac{m_{s}}{\mu_{0}}\left(\left(1+\frac{\alpha}{2}\right) \frac{m_{s}}{\mu_{0}} \frac{p^{2}}{\mu_{0}^{2}+p^{2}}-\frac{\Omega_{0}^{\prime}\left(\mu_{0} / m_{s}\right)}{\Omega_{0}\left(\mu_{0} / m_{s}\right)}\right) .
\end{aligned}
$$

For large values of the linear momentum, $p \gg \mu_{0}$, the instantaneous mass decays over long times, $t \gg 1 / m_{s}$, as follows:

$$
M_{p}(t) \sim p\left(1+\bar{\zeta}_{p}\left(m_{s} t\right)^{-2}\right)
$$

where

$$
\bar{\zeta}_{p}=(1+\alpha) \frac{m_{s}}{\mu_{0}}\left(\left(1+\frac{\alpha}{2}\right) \frac{m_{s}}{\mu_{0}}-\frac{\Omega_{0}^{\prime}\left(\mu_{0} / m_{s}\right)}{\Omega_{0}\left(\mu_{0} / m_{s}\right)}\right) .
$$

If the linear momentum vanishes, $p=0$, the instantaneous mass (at rest) tends over long times, $t \gg 1 / m_{s}$, to the minimum value of the mass spectrum:

$$
M_{0}(\infty)=\mu_{0}
$$

with the following dominant algebraic decay:

$$
M_{0}(t) \sim M_{0}(\infty)\left(1+\zeta_{0}\left(m_{s} t\right)^{-2}\right)
$$

where

$$
\zeta_{0}=-(1+\alpha) \frac{m_{s}}{\mu_{0}} \frac{\Omega_{0}^{\prime}\left(\mu_{0} / m_{s}\right)}{\Omega_{0}\left(\mu_{0} / m_{s}\right)} .
$$

The instantaneous decay rate $\Gamma_{p}(t)$ vanishes over long times, $t \gg 1 / m_{s}$, according to the following dominant algebraic decay:

$$
\Gamma_{p}(t) \sim \frac{2(1+\alpha)}{t} .
$$

Differently from the survival probability and from the instantaneous mass, the dominant asymptotic form of the instantaneous decay rate is independent of the linear momentum $p$. Consequently, the instantaneous decay rate at rest remains approximately unchanged over long times in the reference frame where the unstable particle moves with linear momentum $p$. Notice that decay laws (29) and (31) are in accordance with the ones which are obtained in [23] for a wider class of MDDs. 


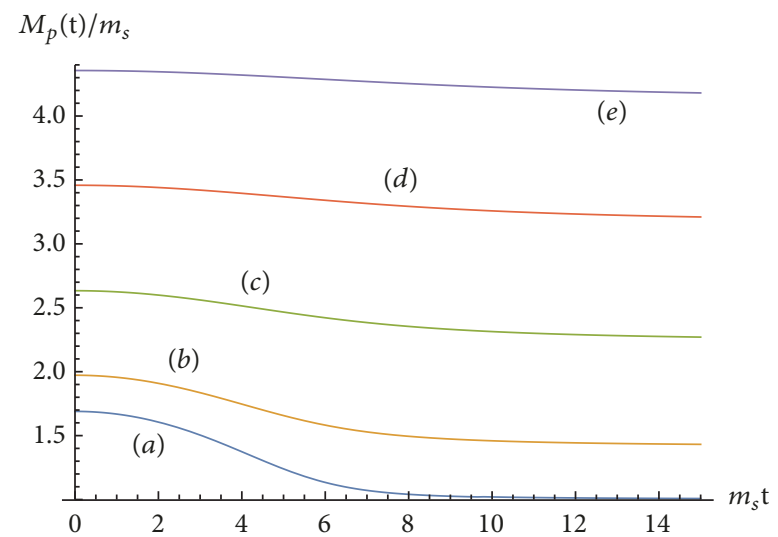

FIGURE 8: (Color online) quantity $M_{p}(t) / m_{s}$ versus $\left(m_{s} t\right)$ for $0 \leq$ $m_{s} t \leq 15$, MDDs given by (21), $\mu_{0}=m_{s}, \alpha=1$, and different values of the linear momentum $p$. Curve $(a)$ corresponds to $p=0 m_{s} ;(b)$ corresponds to $p=m_{s} ;(c)$ corresponds to corresponds to $p=2 m_{s}$; (d) corresponds to $p=3 m_{s} ;(e)$ corresponds to $p=4 m_{s}$.

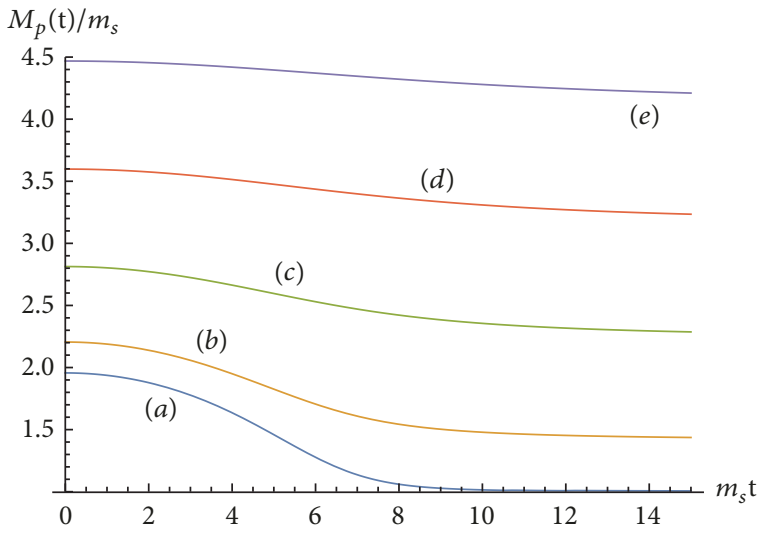

FIGURE 9: (Color online) quantity $M_{p}(t) / m_{s}$ versus $\left(m_{s} t\right)$ for $0 \leq$ $m_{s} t \leq 15$, MDDs given by (21), $\mu_{0}=m_{s}, \alpha=2$, and different values of the linear momentum $p$. Curve $(a)$ corresponds to $p=0 m_{s} ;(b)$ corresponds to $p=m_{s} ;(c)$ corresponds to corresponds to $p=2 m_{s}$; (d) corresponds to $p=3 m_{s}$; $(e)$ corresponds to $p=4 m_{s}$.

Numerical analysis of the instantaneous mass or the instantaneous decay rate is displayed in Figures 8, 9, 12, and 13 or Figures 10, 11, 14, and 15, respectively. The computed MDDs are given by the toy form (21) of the auxiliary function for different values of the nonnegative power $\alpha$ and of the linear momentum $p$. The asymptotic lines of Figure 12 and the asymptotic horizontal lines of Figure 13 are in accordance with the long-time inverse-power-law decays of the instantaneous mass, given by (24) and (29). The asymptotic horizontal lines of Figure 13 agree with (32) and with expression (14) of the scaling factor. The asymptotic lines appearing in Figure 14 and the asymptotic horizontal lines of Figure 15, at ordinate 1, agree with the long-time inversepower-law behavior of the instantaneous decay rate, given by (31).

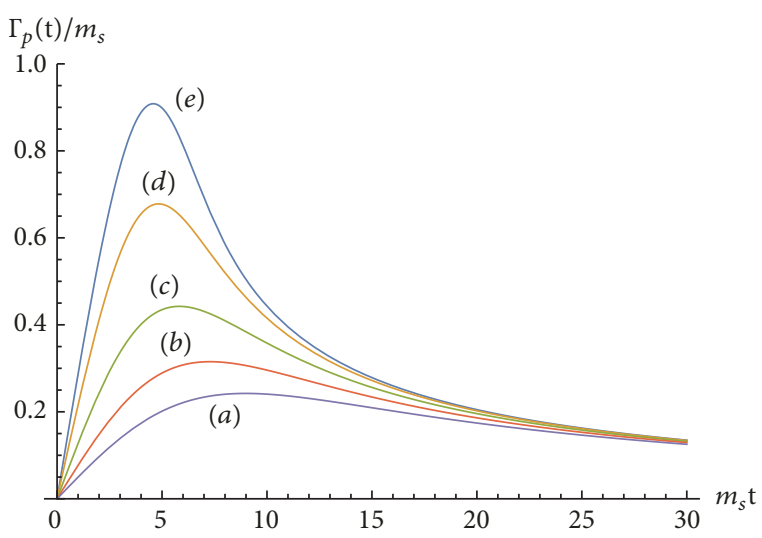

FIGURE 10: (Color online) quantity $\Gamma_{p}(t) / m_{s}$ versus $\left(m_{s} t\right)$ for $0 \leq$ $m_{s} t \leq 30$, MDDs given by (21), $\mu_{0}=m_{s}, \alpha=1$, and different values of the linear momentum $p$. Curve $(a)$ corresponds to $p=4 m_{s}$; (b) corresponds to $p=3 m_{s} ;(c)$ corresponds to $p=2 m_{s} ;(d)$ corresponds to $p=1 m_{s} ;(e)$ corresponds to $p=0 m_{s}$.

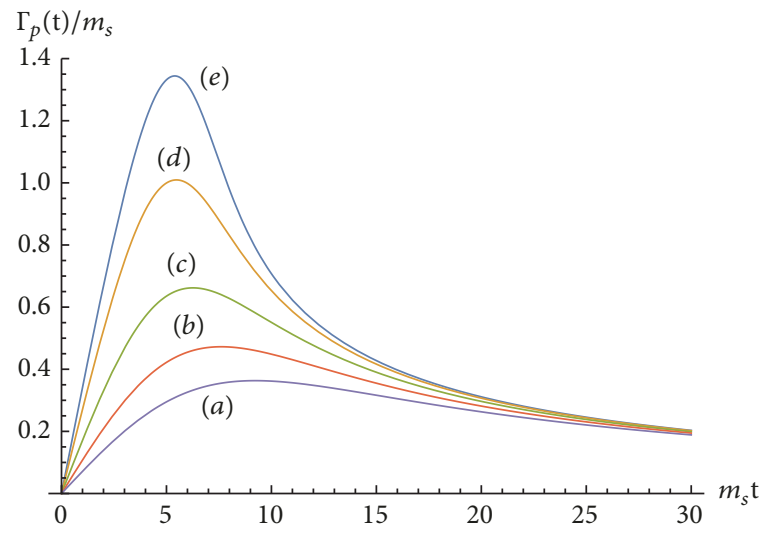

FIGURE 11: (Color online) quantity $\Gamma_{p}(t) / m_{s}$ versus $\left(m_{s} t\right)$ for $0 \leq$ $m_{s} t \leq 30$, MDDs given by (21), $\mu_{0}=m_{s}, \alpha=2$, and different values of the linear momentum $p$. Curve $(a)$ corresponds to $p=4 m_{s}$; (b) corresponds to $p=3 m_{s} ;(c)$ corresponds to $p=2 m_{s} ;(d)$ corresponds to $p=1 m_{s} ;(e)$ corresponds to $p=0 m_{s}$.

\section{Relativistic Time Dilation and Survival Probability}

In Section 2.1 how the survival probability at rest, $\mathscr{P}_{0}(t)$, transforms, due to the relativistic time dilation, in the reference frame where the unstable particle moves with constant linear momentum $p$ is reported. The transformed survival probability, $\mathscr{P}_{p}(t)$, is related to the survival probability at rest, $\mathscr{P}_{0}(t)$, by the scaling law (6). The scaling factor consists in the corresponding relativistic Lorentz factor $\gamma_{L}$.

The analysis performed in Section 3 shows that, for the class of MDDs under study, the survival probability $\mathscr{P}_{p}(t)$ and the survival probability at rest $\mathscr{P}_{0}(t)$ are related by the scaling law (18) over long times. The corresponding scaling factor $\chi_{p}$ is given by (14). It is worth noticing that the scaling factor $\chi_{p}$ coincides with the ratio of the asymptotic form of the instantaneous mass $M_{p}(t)$ and the asymptotic 


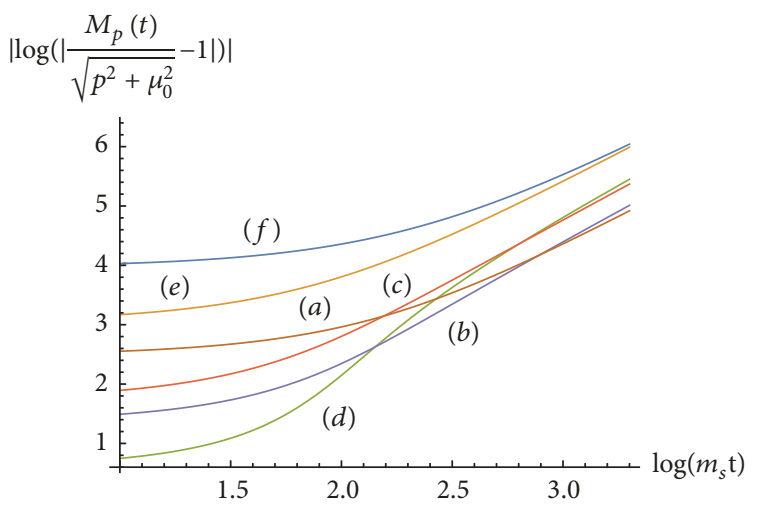

FIGURE 12: (Color online) quantity $\left|\log \left(\left|M_{p}(t) / \sqrt{p^{2}+\mu_{0}^{2}}-1\right|\right)\right|$ versus $\log \left(m_{s} t\right)$ for $e \leq m_{s} t \leq e^{3.3}$, MDDs given by (21), $\mu_{0}=m_{s}$, and different values of the parameter $\alpha$ and of the linear momentum $p$. Curve (a) corresponds to $\alpha=2$ and $p=4 m_{s}$; (b) corresponds to $\alpha=2$ and $p=2 m_{s} ;(c)$ corresponds to $\alpha=1$ and $p=2 m_{s} ;(d)$ corresponds to $\alpha=2$ and $p=m_{s}$; (e) corresponds to $\alpha=0$ and $p=3 m_{s} ;(f)$ corresponds to $\alpha=0$ and $p=5 m_{s}$.

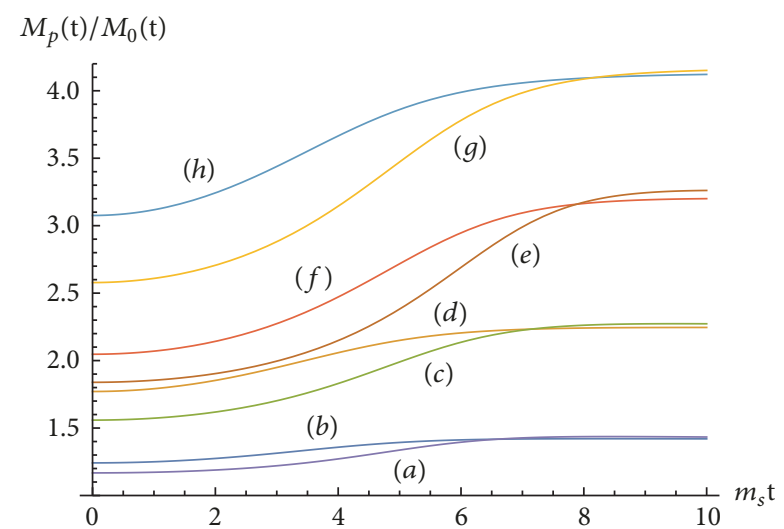

FIGURE 13: (Color online) ratio $M_{p}(t) / M_{0}(t)$ versus $\left(m_{s} t\right)$ for $0 \leq$ $m_{s} t \leq 10$, MDDs given by (21), $\mu_{0}=m_{s}$, and different values of the parameter $\alpha$ and of the linear momentum $p$. Curve $(a)$ corresponds to $\alpha=1$ and $p=m_{s} ;(b)$ corresponds to $\alpha=0$ and $p=m_{s} ;(c)$ corresponds to $\alpha=1$ and $p=2 m_{s} ;(d)$ corresponds to $\alpha=0$ and $p=2 m_{s} ;(e)$ corresponds to $\alpha=2$ and $p=3 m_{s} ;(f)$ corresponds to $\alpha=1$ and $p=3 m_{s} ;(g)$ corresponds to $\alpha=1$ and $p=4 m_{s} ;(h)$ corresponds to $\alpha=0$ and $p=4 m_{s}$.

expression of the instantaneous mass at rest $M_{0}(t)$ of the moving unstable particle:

$$
\chi_{p}=\frac{M_{p}(\infty)}{M_{0}(\infty)} .
$$

At this stage, consider the reference frame $\mathfrak{S}$ where a mass at rest which is equal to the asymptotic value $M_{0}(\infty)$ becomes $M_{p}(\infty)$ due to the relativistic transformation of the mass. According to (32), in the reference frame $\mathbb{S}$ the corresponding relativistic Lorentz factor coincides with the scaling factor $\chi_{p}$. We remind that, initially, the unstable quantum system is not in an eigenstate of the Hamiltonian. Consequently, in the present model the mass of the unstable

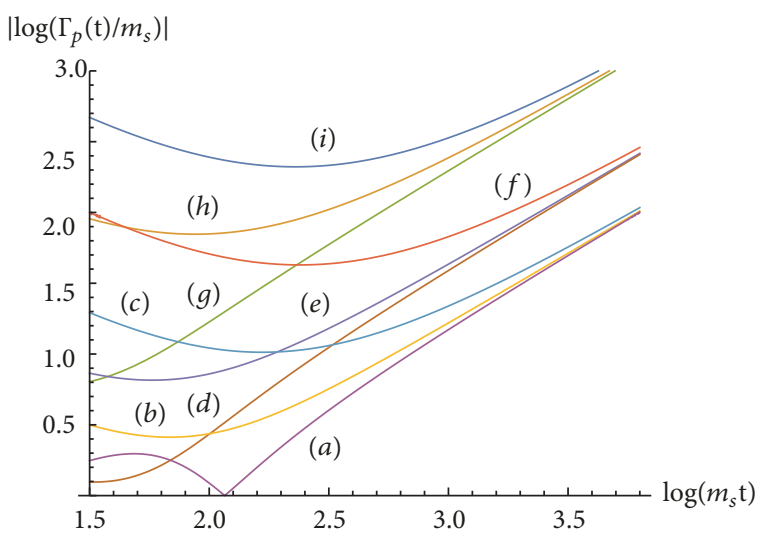

FIGURE 14: (Color online) quantity $\left|\log \left(\Gamma_{p}(t) / m_{s}\right)\right|$ versus $\log \left(m_{s} t\right)$ for $e^{1.5} \leq m_{s} t \leq e^{3.8}$, MDDs given by (21), $\mu_{0}=m_{s}$, and different values of the parameter $\alpha$ and of the linear momentum $p$. Curve (a) corresponds to $\alpha=2$ and $p=0 m_{s} ;(b)$ corresponds to $\alpha=2$ and $p=2 m_{s} ;(c)$ corresponds to $\alpha=2$ and $p=4 m_{s} ;(d)$ corresponds to $\alpha=1$ and $p=0 m_{s} ;(e)$ corresponds to $\alpha=1$ and $p=2 m_{s} ;(f)$ corresponds to $\alpha=1$ and $p=5 m_{s} ;(g)$ corresponds to $\alpha=0$ and $p=0 m_{s} ;(h)$ corresponds to $\alpha=0$ and $p=3 m_{s} ;(i)$ corresponds to $\alpha=0$ and $p=5 m_{s}$.

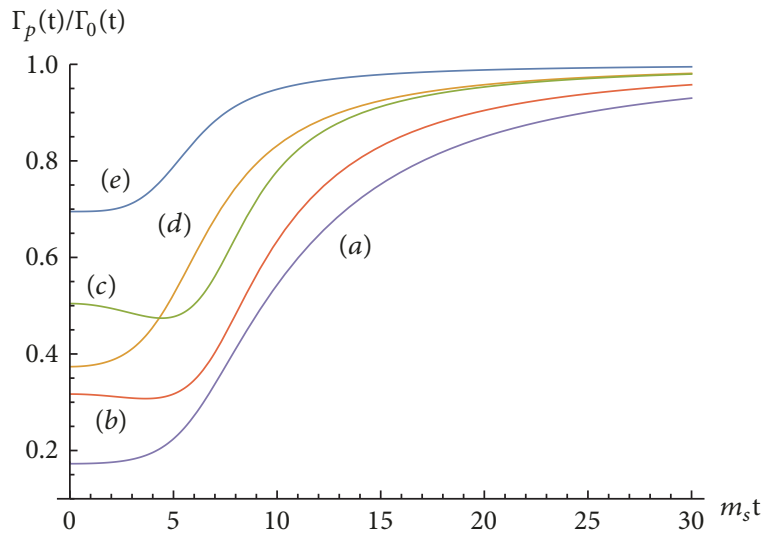

FIGURE 15: (Color online) ratio $\Gamma_{p}(t) / \Gamma_{0}(t)$ versus $\left(m_{s} t\right)$ for $0 \leq$ $m_{s} t \leq 30$, MDDs given by (21), $\mu_{0}=m_{s}$, and different values of the parameter $\alpha$ and of the linear momentum $p$. Curve (a) corresponds to $\alpha=1$ and $p=4 m_{s} ;(b)$ corresponds to $\alpha=2$ and $p=3 m_{s} ;(c)$ corresponds to $\alpha=2$ and $p=2 m_{s} ;(d)$ corresponds to $\alpha=0$ and $p=2 m_{s} ;(e)$ corresponds to $\alpha=0$ and $p=m_{s}$.

particle is not defined. On the contrary, the instantaneous mass is properly defined in terms of the survival amplitude. See $[14,16,17]$ for details.

In light of the above observations, the long-time scaling law (18) can be interpreted as an effect of the relativistic time dilation if the asymptotic value $M_{p}(\infty)$ of the instantaneous mass is considered to be the effective mass of the unstable particle over long times. In fact, in the reference frame $\mathbb{\Xi}$ the mass at rest $M_{0}(\infty)$, which is equal to the value $\mu_{0}$, moves with linear momentum $p$, or, equivalently, with constant velocity $1 / \sqrt{1+\mu_{0}^{2} / p^{2}}$, and becomes the relativistic mass $M_{p}(\infty)$, which is equal to the value $\sqrt{\mu_{0}^{2}+p^{2}}$. Concurrently, 
in the reference frame $\mathbb{S}$ the survival probability at rest $\mathscr{P}_{0}(t)$ transforms, according to the relativistic time dilation, in the survival probability $\mathscr{P}_{p}(t)$ and obeys the scaling law (6), or, equivalently, (18), over long times. In this context, the crucial condition of nonvanishing lower bound of the mass spectrum, $\mu_{0} / m_{s}>0$, suggests that the long-time relativistic dilation and the scaling law (6), or, equivalently, (18), hold uniquely for an unstable moving particle with nonvanishing effective mass, $M_{0}(\infty)>0$.

\section{Summary and Conclusions}

The relativistic quantum decay laws of a moving unstable particle have been analyzed over short and long times for an arbitrary value $p$ of the (constant) linear momentum. The MDDs under study exhibit power-law behaviors near the (nonvanishing) lower bound $\mu_{0}$ of the mass spectrum. Due to the arbitrariness of the linear momentum, the ultrarelativistic and nonrelativistic limits have been obtained as particular cases.

The survival probability, which is detected in the rest reference frame of the unstable particle, transforms in the reference frame where the unstable particle moves with linear momentum $p$, approximately according to a scaling law, over long times. The scaling factor is determined by the lower bound $\mu_{0}$ of the mass spectrum and by the linear momentum $p$ of the particle. The scaling law can be interpreted as the effect of the relativistic time dilation if the asymptotic form of the instantaneous mass $M_{p}(t)$ is considered as the effective mass of the moving unstable particle over long times. In fact, consider the reference frame $\mathfrak{S}$ where a mass at rest of magnitude $\mu_{0}$ moves with velocity $1 / \sqrt{1+\mu_{0}^{2} / p^{2}}$, or, equivalently, with linear momentum $p$. The mass at rest $\mu_{0}$ coincides with the asymptotic value of the instantaneous mass at rest, $M_{0}(\infty)$, of the moving unstable particle. In the reference frame $\mathfrak{S}$ the transformed mass, which is equal to the value $\sqrt{\mu_{0}^{2}+p^{2}}$, coincides with the asymptotic value $M_{p}(\infty)$ of the instantaneous mass of the particle. Simultaneously, in the reference frame $\mathbb{S}$ the dilation of times, which is suggested by the special relativity, transforms the survival probability at rest according to the mentioned scaling law. The above description indicates the value $1 / \sqrt{1+\mu_{0}^{2} / p^{2}}$ as the asymptotic velocity of the moving unstable particle.

We stress that the present interpretation is an attempt to ascribe the transformation laws of the long-time survival probability to the dilation of times which is provided by the theory of special relativity. However, a clear scaling transformation of the survival probability at rest holds, approximately over long times, if the decay is observed in the reference frame where the unstable particle moves with constant linear momentum. The scaling law can still be interpreted as the effect of a time dilation which appears by changing reference frame. The dilation is determined uniquely by the scaling factor which depends on the mass spectrum and on the dynamics of the unstable particle. The theoretical results are confirmed by the numerical analysis.
While the instantaneous mass transforms by changing reference frame, no transformation is found, approximately, for the instantaneous decay rate over long times. In fact, the instantaneous decay rate vanishes, over long times, approximately independently of the linear momentum of the moving particle. Consequently, the long-time instantaneous decay rate is approximately invariant by changing reference frame.

In conclusion, the present analysis shows further ways to describe the long-time transformations of the decay laws of moving unstable particles in terms of model-independent properties of the mass spectrum. The role of the (nonvanishing) mass at rest in the relativistic transformation is assumed in the present description by the (nonvanishing) lower bound of the mass spectrum.

\section{Data Availability}

The results of this article are entirely theoretical and analytical. For each result, the main steps of the corresponding demonstration are reported in the text. The article is fully consistent without the support of any additional data.

\section{Conflicts of Interest}

The author declares that there are no conflicts of interest regarding the publication of this paper.

\section{References}

[1] L. A. Khalfin, "Quantum Theory of Unstable Particles and Relativity," Tech. Rep. PDMI PREPRINT-6/1997, St. Petersburg Department of Stelkov Mathematical Institute, St. Ptersburg, Russia, 1997.

[2] L. Fonda, G. C. Ghirardi, and A. Rimini, "Decay theory of unstable quantum systems," Reports on Progress in Physics, vol. 41, no. 4, pp. 587-631, 1978.

[3] B. Bakamjian, "Relativistic particle dynamics," Physical Review A: Atomic, Molecular and Optical Physics, vol. 121, no. 6, pp. 1849-1851, 1961.

[4] F. Coester and W. N. Polyzou, "Relativistic quantum mechanics of particles with direct interactions," Physical Review D: Particles, Fields, Gravitation and Cosmology, vol. 26, no. 6, pp. 13481367, 1982.

[5] P. Exner, "Representations of the Poincare group associated with unstable particles," Physical Review D: Particles, Fields, Gravitation and Cosmology, vol. 28, no. 10, pp. 2621-2627, 1983.

[6] E. V. Stefanovich, Relativistic Quantum Theory of Particles, vol. 1 and 2, Lambert Academic, 2015.

[7] E. V. Stefanovich, "Quantum effects in relativistic decays," International Journal of Theoretical Physics, vol. 35, no. 12, pp. 2539-2554, 1996.

[8] M. Shirokov, "Decay law of moving unstable particle," International Journal of Theoretical Physics, vol. 43, no. 6, pp. 1541-1553, 2004.

[9] D. H. Frisch and J. H. Smith, "Measurement of the relativistic time dilation using $\mu$-mesons," American Journal of Physics, vol. 31, no. 5, article 342, 1963. 
[10] J. Bailey, K. Borer, F. Combley et al., "Measurements of relativistic time dilatation for positive and negative muons in a circular orbit," Nature, vol. 268, no. 5618, pp. 301-305, 1977.

[11] F. J. M. Farley, “The CERN (g-2) measurements," Zeitschrift für Physik C Particles and Fields, vol. 56, supplement 1, pp. S88-S96, 1992.

[12] M. I. Shirokov, "Evolution in time of moving unstable systems," Concepts of Physics, vol. 3, pp. 193-205, 2006.

[13] M. I. Shirokov, "Moving system with speeded-up evolution," Physics of Particles and Nuclei Letters, vol. 6, no. 1, pp. 14-17, 2009.

[14] K. Urbanowski, "Decay law of relativistic particles: quantum theory meets special relativity," Physics Letters. B. Particle Physics, Nuclear Physics and Cosmology, vol. 737, pp. 346-351, 2014.

[15] F. Giacosa, "Decay law and time dilatation," Acta Physica Polonica B, vol. 47, no. 9, pp. 2135-2150, 2016.

[16] K. Urbanowski, "On the velocity of moving relativistic unstable quantum systems," Advances in High Energy Physics, vol. 2015, Article ID 461987, 2015.

[17] K. Urbanowski, "Non-classical behavior of moving relativistic unstable particles," Jagellonian University. Institute of Physics. Acta Physica Polonica B, vol. 48, no. 8, pp. 1411-1432, 2017.

[18] W. M. Gibson and B. R. Pollard, Symmetry Principles in Elementary Particle Physics, Cambridge, UK, 1976.

[19] M. L. Goldberger and K. M. Watson, Collision Theory, Wiley, New York, NY, USA, 1964.

[20] K. Urbanowski, "General properties of the evolution of unstable states at long times," The European Physical Journal D, vol. 54, no. 1, pp. 25-29, 2009.

[21] K. Urbanowski, "Long time properties of the evolution of an unstable state," Open Physics, vol. 7, no. 4, pp. 696-703, 2009.

[22] F. Giraldi, "Logarithmic decays of unstable states," The European Physical Journal D, vol. 69, no. 1, 2015.

[23] F. Giraldi, "Logarithmic decays of unstable states II," The European Physical Journal D, vol. 70, no. 11, article no. 229, 2016.

[24] K. Urbanowski, "Early-time properties of quantum evolution," Physical Review A: Atomic, Molecular and Optical Physics, vol. 50, pp. 2847-2853, 1994.

[25] C. Møller, The theory of relativity, Clarendon Press, Oxford, UK, 1972.

[26] A. Erdélyi, Asymptotic Expansions, Dover, New York, NY, USA, 1956.

[27] R. Wong, Asymptotic Approximations of Integrals, Academic Press, Boston, Mass, USA, 1989. 

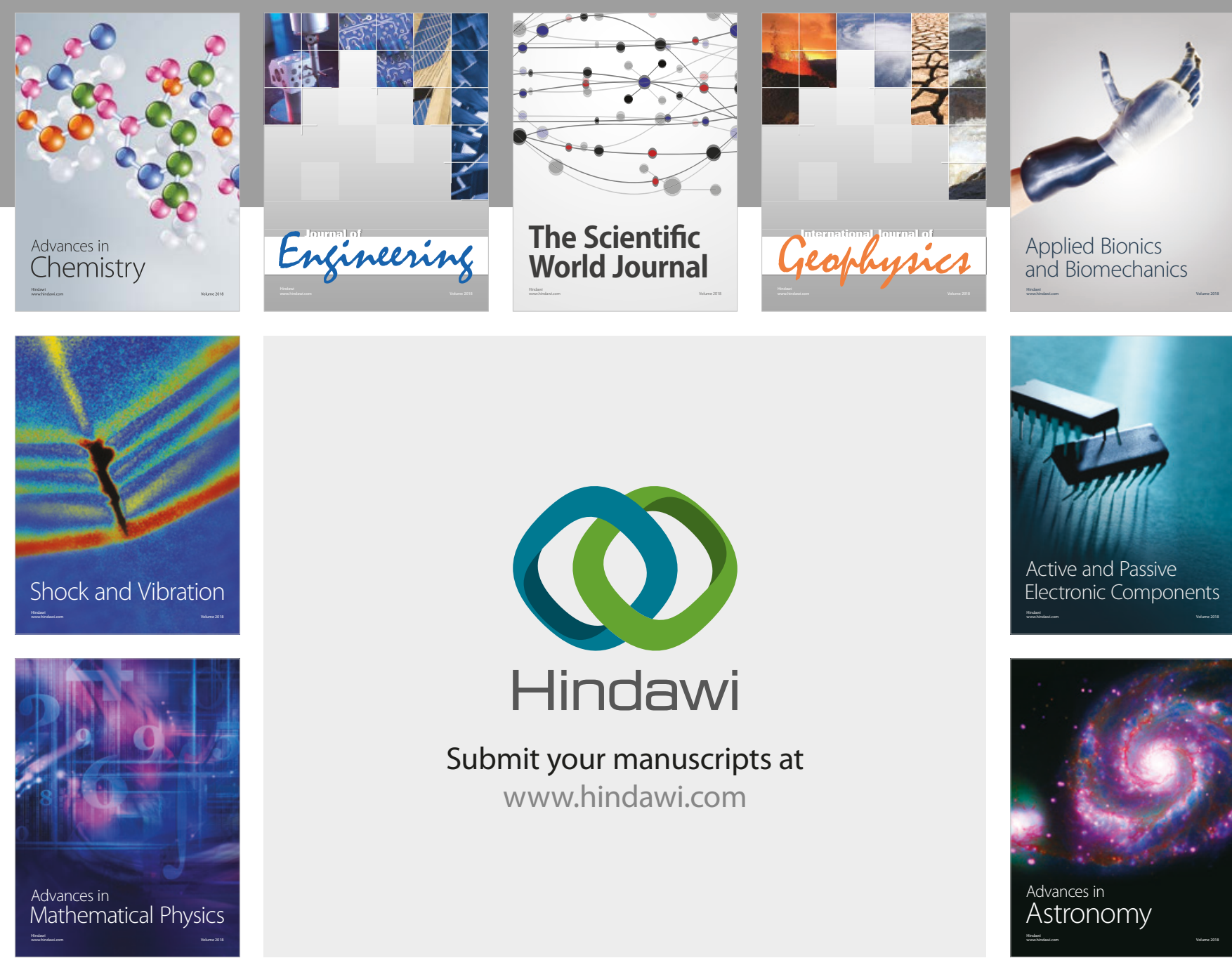

Submit your manuscripts at

www.hindawi.com

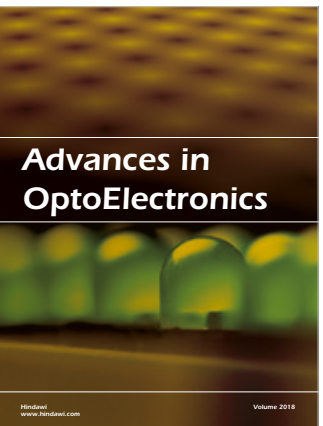

\section{Rotcting Machinery}
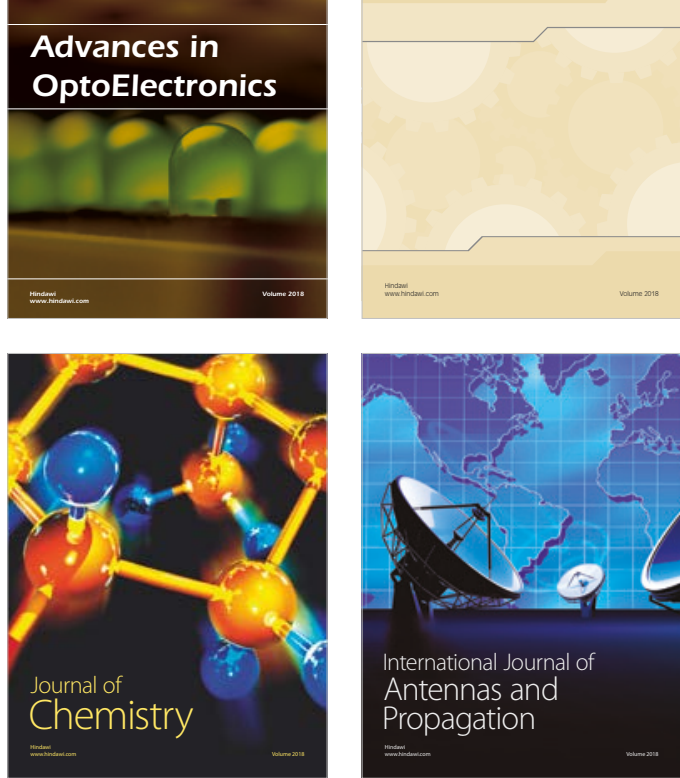

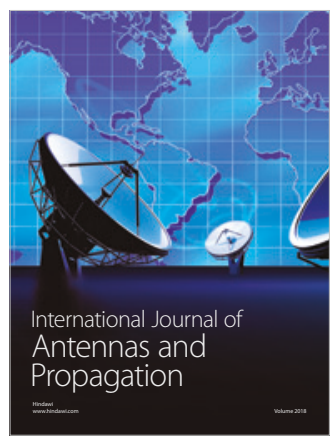

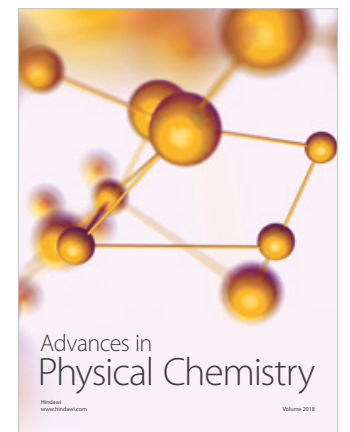

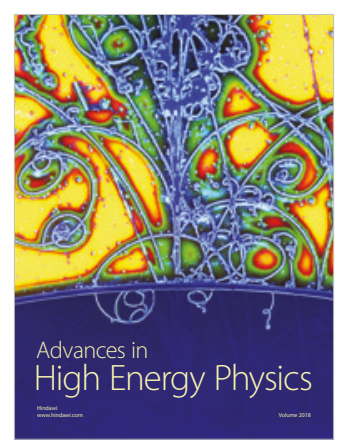

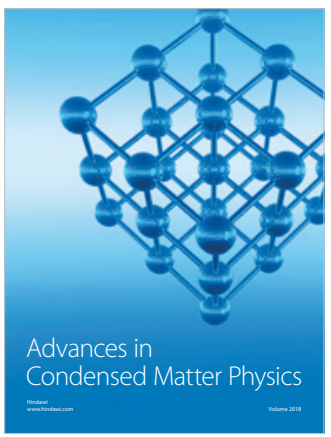

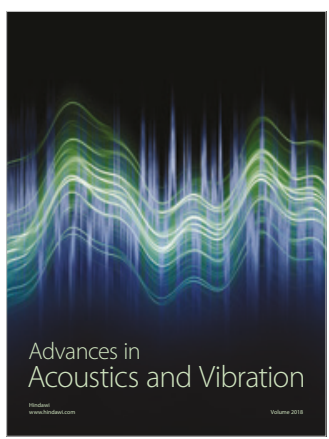

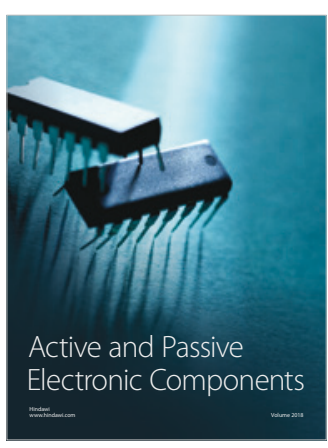
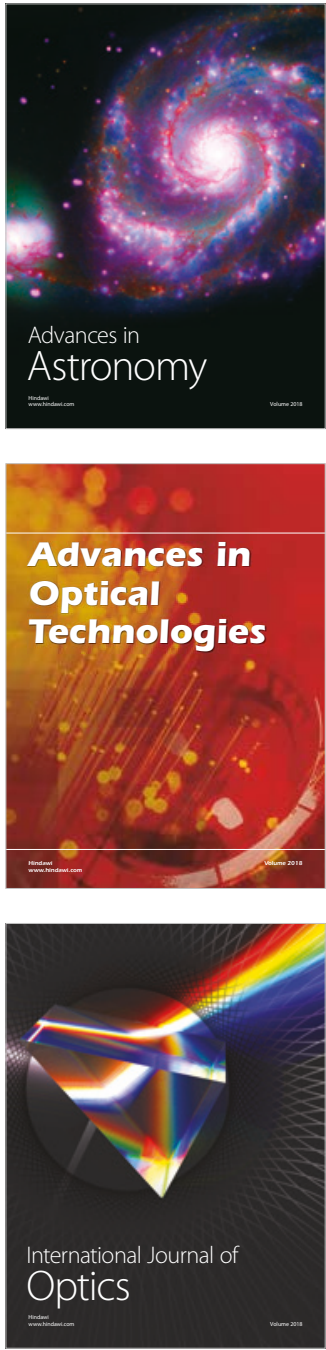